\title{
On the equations of mathematical hydraulics
}

By S. J. Jacobs, Dept of Atmospheric, Oceanic, and Space Sciences, The University of Michigan, Ann Arbor, USA

\section{Introduction}

We will be concerned here with turbulent two-dimensional open channel flow down a plane inclined at a constant angle to the horizontal, as shown in Fig. 1. In the hydraulics literature flows of this type are treated using two different theories, which apparently have little in common.

According to the simpler of the theories, classical mathematical hydraulics [1], the fluid depth $h(x, t)$ and the depth-averaged velocity $\tilde{u}(x, t)$ solve the equations

$$
\begin{aligned}
& \frac{\partial h}{\partial t}+\frac{\partial(h \bar{u})}{\partial x}=0, \\
& \frac{\partial \bar{u}}{\partial t}+\ddot{u} \frac{\partial \bar{u}}{\partial x}+g^{\prime} \frac{\partial h}{\partial x}=g^{\prime} \tan \alpha-C_{D} \frac{|\bar{u}| \bar{u}}{h},
\end{aligned}
$$

where $x$ is distance measured parallel to the bottom, $t$ is time, $\alpha$ is the angle between the channel bed and the horizontal, $g^{\prime}=g \cos \alpha$, and $C_{D}$ is the bottom drag coefficient. In this theory the flow is governed by (1.1) and (1.2) except at discontinuities of the dependent variables, where, letting $[f]$ denote the discontinuity in a quantity $f$ at $x=s(t), h$ and $\bar{u}$ satisfy the jump conditions

$$
[h \bar{u}]=[h] \frac{d s}{d t}, \quad\left[h \bar{u}^{2}+\frac{1}{2} g^{\prime} h^{2}\right]=[h \bar{u}] \frac{d s}{d t} .
$$

If the bottom drag term in (1.2) is omitted, the theory described by the differential equations (1.1)-(1.2) and the jump conditions (1.3) reduces to the standard long wave model for free surface flows, which can be shown to be valid on the basis of a scaling argument [1] if the horizontal length scale of the flow is large compared to the vertical scale. Both the inclusion of bottom drag on the right side of (1.2) and its quadratic dependence on the velocity, however, are based on dimensional analysis and observations rather than any deeper theory. It appears, then, that the equations of mathematical hydraulics are in some sense an approximation, but, because 


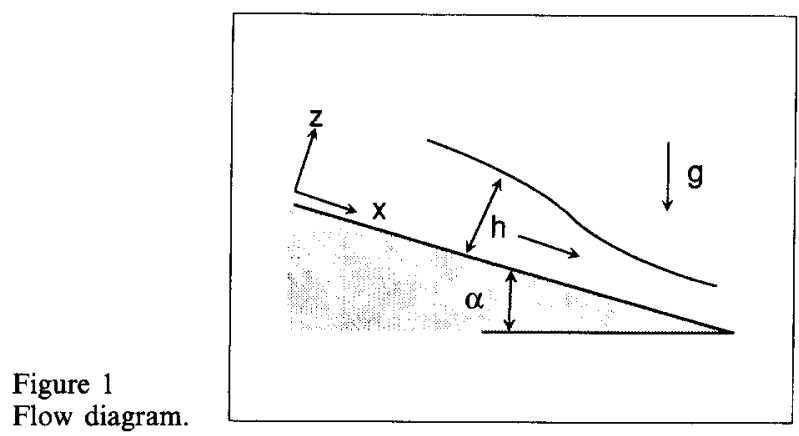

of the absence of any real derivation of these equations in the literature, the nature of the approximation is obscure.

In the more recent class of theories reviewed in [2], fluid motion in an open channel is treated as a turbulent shear flow in which the Reynolds averaged velocity components $(u, w)$ and the Reynolds averaged pressure $p$ depend on distance $z$ from the bottom as well as on $x$ and $t$. When supplemented by boundary and initial conditions and by constitutive equations relating the Reynolds stresses to the mean velocity, the Reynolds averaged continuity and momentum equations provide a complete turbulence model. Examples of numerical calculations using such models and of comparisons of computed velocity profiles with observations are given in [2] and elswhere in the literature.

Our aim here is to reconcile these theories by using asymptotic methods [3] to show that the equations of mathematical hydraulics can be derived from the Reynolds averaged equations for flows which vary sufficiently slowly in the longitudinal direction. Because the hydraulics equations and the equations describing present turbulence models are based on ad hoc assumptions, the derivation serves to increase confidence in both approaches and to provide guidelines for the user of the hydraulics approximation.

To determine the parameter range of interest, assume that Eqs. (1.1)(1.2) provide a first approximation to the governing equations, and let $V$ denote the characteristic longitudinal velocity scale for a flow with longitudinal length scale $L_{0}$ and characteristic depth $H_{0}$. Because classical hydraulics assumes that the leading term in the solution for the longitudinal velocity is independent of $z$, it follows that the aspect ratio $\delta=H_{0} / L_{0}$ is small compared to unity, that transients take the form of long gravity waves with characteristic frequency $C_{0} / L_{0}$, where $C_{0}=\left(g^{\prime} H_{0}\right)^{1 / 2}$, and that the ratio of the advective term $\bar{u}(\partial \bar{u} / \partial x)$ in (1.2) to the time derivative $\partial \vec{u} / \partial t$ is of the order of the Froude number $F=V / C_{0}$. Weak flows, for which $F \ll 1$, are treated in [4], where it is shown that the free surface elevation for weakly nonlinear flow satisfies a damped version of the Korteweg de Vries 
equation. We will be concerned here with large amplitude motions, and so we restrict our attention to flows for which $F \geq O(1)$.

In this case the material derivative $D \bar{u} / D t$ on the left side of (1.2) is of order $V^{2} / L_{0}$, while the bottom stress term on the right side of the equation is of order $C_{D}\left(V^{2} / H_{0}\right)$, where $C_{D}$ is the drag coefficient introduced earlier. If the bottom stress term is large compared to $D \bar{u} / D t$, the flow is described by the kinematic wave theory for open channel flow treated in [1]. Since it is clear that turbulence effects are unimportant if the bottom stress term is small compared to $D \bar{u} / D t$, the most general theory is obtained by assuming a balance between these terms. This scaling argument therefore suggests that the hydraulics approximation is valid if the Froude number is of order unity or larger and if $\delta$ and $C_{D}$ are of comparable magnitude.

Defining the bottom friction velocity $u_{*}$ in the usual way, $\kappa=0.4$ as the Karman constant, and $z_{0}$ as the bottom roughness length, and noting that in open channel flow the logarithmic velocity profile

$$
u=\frac{u_{*}}{\kappa} \ln \left(\frac{z}{z_{0}}\right)
$$

provides a fairly accurate representation for the velocity throughout most of the water column, it can be seen that $u_{*}$ and the velocity scale $V$ are related by $u_{*} / V=O(\beta)$, where

$$
\beta=\frac{\kappa}{\ln \left(H_{0} / z_{0}\right)} .
$$

Therefore, since $\left(u_{*} / V\right)^{2}=O\left(C_{D}\right)$, the drag coefficient is of the order $\beta^{2}$, and the conditions for which classical hydraulics can be expected to provide an accurate approximation in the treatment of open channel flows are summarized by

$$
F \geq O(1), \quad \delta \ll O(1), \quad \delta=O\left(\beta^{2}\right),
$$

where $\beta$ is given by (1.4). Typical magnitudes of this parameter are of order $10^{-1}$ or smaller, and therefore the last two relations in (1.5) are consistent.

The flow is treated here for the parameter range given by (1.5) using Donaldson's second order turbulence model, as described in [5]. Section 2 gives the equations of the model in dimensionless form, and Section 3 a derivation of a version of (1.1) and (1.2) in which an expression for the drag coefficient as a function of the bottom roughness length is determined. In Section 4 the jump conditions (1.3) are verified using approximate equations governing the bore structure, and it is inferred that classical hydraulics provides an acceptable approximation to the flow outside bore regions for the parameter range considered in the theory. 


\section{Formulation}

Temporarily using subscript notation and letting $f_{i}$ denote the body force per unit mass, the equations of Donaldson's turbulence model describing flow of a fluid with constant density $\varrho$ can be expressed in the form

$$
\begin{aligned}
& \frac{\partial u_{k}}{\partial x_{k}}=0, \\
& \frac{D u_{i}}{D t}=-\frac{1}{\varrho} \frac{\partial p}{\partial x_{i}}+\frac{\partial \tau_{k i}}{\partial x_{k}}+f_{i}, \\
& \frac{D \tau_{i j}}{D t}+\pi_{i j}=c_{1} \frac{\partial}{\partial x_{k}}\left(q \Lambda \frac{\partial \tau_{i j}}{\partial x_{k}}\right)-\frac{q}{\Lambda}\left(\tau_{i j}+\frac{q^{2}}{4} \delta_{i j}\right) . \\
& \frac{D q^{2}}{D t}=\pi_{k k}+c_{1} \frac{\partial}{\partial x_{k}}\left(q \Lambda \frac{\partial q^{2}}{\partial x_{k}}\right)-\frac{q^{3}}{4 \Lambda}, \\
& \frac{D \Lambda}{D t}=c_{1} \frac{\partial}{\partial x_{k}}\left(q \Lambda \frac{\partial \Lambda}{\partial x_{k}}\right)+c_{2} q-c_{3} \frac{\Lambda}{2 q^{2}} \pi_{k k}-\frac{c_{4}}{q} \frac{q(q \Lambda)}{\partial x_{k}} \frac{\partial(q \Lambda)}{\partial x_{k}} .
\end{aligned}
$$

In these equations $u_{i}$ is the component of Reynolds averaged velocity in the $x_{i}$ direction, $p$ is the Reynolds averaged gauge pressure, $\tau_{i j}$ is the ratio of the Reynolds stress tensor to the fluid density, $q^{2} / 2$ is the turbulent kinetic energy per unit mass, $\Lambda$ is a dissipation length scale, $D / D t$ is the material derivative operator, $\delta_{i j}$ is the Kronecker delta, and the turbulent work tensor $\pi_{i j}$ is defined by

$$
\pi_{i k}=\tau_{i n} \frac{\partial u_{k}}{\partial x_{n}}+\tau_{k n} \frac{\partial u_{i}}{\partial x_{n}}
$$

The constants in the field equations and an additional constant $c_{5}$ are given by

$$
\begin{aligned}
& c_{1}=0.3, \quad c_{2}=0.04375+(0.15 \sqrt{2}) \kappa^{2}, \\
& c_{3}=0.35, \quad c_{4}=0.375, \quad c_{5}=2^{3 / 4} \kappa,
\end{aligned}
$$

where, as before, $\kappa=0.4$ is the Karman constant.

It is convenient to use dyadic notation in expressing the boundary conditions at a material surface. Assume that the unit normal $\hat{n}$ to the surface has a positive vertical component, and consider first the case for which the traction has nonvanishing perpendicular and tangential components. Let $t_{n}$ and $t_{t}$ denote the perpendicular and tangential parts of the traction stress exerted on the surface by the material above it, $\boldsymbol{u}$ the fluid velocity, $\boldsymbol{u}_{t}$ the tangential speed of the surface, $n$ the distance from the surface along $\hat{n}, z_{0}$ the surface roughness length, $\vec{I}$ the unit tensor, and $\vec{\tau}$ the 
kinematic Reynolds stress tensor, and define the vector friction velocity $s$ by $t_{t}=\rho|s| s$. Using these definitions, the boundary conditions become

$$
\begin{array}{ll}
\boldsymbol{u}-(\hat{n} \cdot \boldsymbol{u}) \hat{n} \rightarrow \boldsymbol{u}_{t}+\frac{n}{|n|} \frac{s}{\kappa} \ln \left(\frac{|n|}{z_{0}}\right), & \frac{D n}{D t} \rightarrow 0, \\
\overrightarrow{\boldsymbol{\tau}} \rightarrow|s|(\hat{n} \boldsymbol{s}+s \hat{n})-2^{1 / 2}\left(|s|^{2} \vec{I}+s s\right), & p \rightarrow-\rho\left(t_{n}+2^{1 / 2}|s|^{2}\right),
\end{array}
$$

and

$$
q \rightarrow 2^{5 / 4}|s|, \quad \Lambda \rightarrow c_{5}|n|,
$$

as $n \rightarrow 0$. Here $n /|n|$ is positive or negative depending on whether the flow takes place above or below the surface, respectively, and it can be verified that Eqs. (2.8) $-(2.10)$ provide an asymptotic solution to the equations of the model valid in the limit $n \rightarrow 0$.

The boundary conditions at a surface with vanishing tangential stress will be taken here as

$$
\begin{aligned}
& \tau_{13}^{\prime}=\tau_{23}^{\prime}=0, \quad p=\varrho\left(\tau_{33}^{\prime}-t_{n}\right), \quad \frac{D n}{D t}=0, \\
& \frac{\partial}{\partial n}\left\{\tau_{11}^{\prime}, \tau_{22}^{\prime}, \tau_{33}^{\prime}, \tau_{12}^{\prime}, q, \Lambda\right\}=0,
\end{aligned}
$$

where the stress components are defined with respect to moving rectangular axes $\left(x_{1}^{\prime}, x_{2}^{\prime}, x_{3}^{\prime}\right)$, with $x_{1}^{\prime}$ and $x_{2}^{\prime}$ parallel to the surface. The second set of conditions in (2.11) is based on the assumption that the surface can be treated locally as a symmetry plane if the tangential components of the traction stress vanish, and differs slightly from a set of boundary conditions for this case suggested on p. 46 of [2]. Use of this alternative set of conditions decreases the value of $\Lambda$ at the surface below the magnitude it takes when (2.11) is imposed, but has a comparatively minor effect on the velocity components.

In the problem of interest here, two-dimensional flow in the $\left(x_{1}, x_{3}\right)$ plane over a bottom inclined at an angle $\alpha$ to the horizontal, the body force per unit mass $f_{i}$ has components $f_{1}=g^{\prime} \tan \alpha, f_{2}=0$, and $f_{3}=g^{\prime}$, where $g^{\prime}=g \cos \alpha$. To treat the problem, let $x_{3}=h\left(x_{1}, t\right)$ denote the free surface and use $(x, z)$ as coordinates in place of $\left(x_{1}, x_{3}\right)$. The governing equations for this flow can be expressed in dimensionless form by letting $H_{0}$ and $L_{0}$ denote characteristic values for the fluid depth and longitudinal length and by defining a friction velocity scale $u_{\tau}$ and the longitudinal scale $V$ through

$$
u_{\tau}=\left(g^{\prime} H_{0} \tan \alpha\right)^{1 / 2}, \quad V=\frac{u_{\tau}}{\kappa} \ln \left(\frac{H_{0}}{z_{0}}\right)
$$


The scaled variables are defined by

$$
\begin{aligned}
& x^{*}=\frac{x}{L_{0}}, \quad z^{*}=\frac{z}{H_{0}}, \quad t^{*}=\frac{V t}{L_{0}}, \quad u^{*}=\frac{u}{V}, \quad w^{*}=\frac{L_{0} w}{H_{0} V}, \\
& p^{*}=\frac{p+\rho g^{\prime} z}{\varrho V^{2}}, \quad h^{*}=\frac{h}{H_{0}}, \\
& \tau_{i j}^{*}=\frac{\tau_{i j}}{\left(u_{\tau}\right)^{2}}, \quad q^{*}=\frac{q}{u_{\tau}}, \quad s^{*}=\frac{s}{u_{\tau}}, \quad \Lambda^{*}=\frac{\Lambda}{H_{0}}, \quad \pi_{i j}^{*}=\frac{H_{0} \pi_{i j}}{\left(u_{\tau}\right)^{2} V},
\end{aligned}
$$

where $s$ is the component of the friction velocity in the $x$ direction. Then, repeating the definitions

$$
\delta=\frac{H_{0}}{L_{0}}, \quad \beta=\frac{\kappa}{\ln \left(H_{0} / z_{0}\right)}, \quad F=\frac{V}{\left(g^{\prime} H_{0}\right)^{1 / 2}},
$$

given earlier, and omitting the asterisks, the field equations needed to compute the velocity take the dimensionless form

$$
\begin{aligned}
& \frac{\partial u}{\partial x}+\frac{\partial w}{\partial z}=0, \\
& \frac{D u}{D t}+\frac{\partial p}{\partial x}=\frac{\beta^{2}}{\delta}\left(\delta \frac{\partial \tau_{x x}}{\partial x}+\frac{\partial \tau_{x z}}{\partial z}+1\right), \\
& \delta^{2} \frac{D w}{D t}+\frac{\partial p}{\partial z}=\beta\left(\delta \frac{\partial \tau_{x z}}{\partial x}+\frac{\partial \tau_{z z}}{\partial z}\right), \\
& \delta \frac{D \tau_{x x}}{D t}+\pi_{x x}=\beta\left\{c_{1} \Delta\left(\tau_{x x}\right)-\frac{q}{\Lambda}\left(\tau_{x x}+\frac{q^{2}}{4}\right)\right\}, \\
& \delta \frac{D \tau_{x z}}{D t}+\pi_{x z}=\beta\left\{c_{1} \Delta\left(\tau_{x z}\right)-\frac{q}{\Lambda} \tau_{x z}\right\}, \\
& \delta \frac{D \tau_{z z}}{D t}+\pi_{z z}=\beta\left\{c_{1} \Delta\left(\tau_{z z}\right)-\frac{q}{\Lambda}\left(\tau_{z z}+\frac{q^{2}}{4}\right)\right\}, \\
& \delta \frac{D q^{2}}{D t}=\pi_{x x}+\pi_{z z}+\beta\left\{c_{1} \Delta\left(q^{2}\right)-\frac{q^{3}}{4 \Lambda}\right\}, \\
& \delta \frac{D \Lambda}{D t}+\frac{c_{3} \Lambda}{2 q^{2}}\left(\pi_{x x}+\pi_{z z}\right)=\beta\left\{c_{1} \Delta(\Lambda)+c_{2} q-\frac{c_{4}}{q} R\right\} .
\end{aligned}
$$

In these equations

$$
\frac{D}{D t}=\frac{\partial}{\partial t}+u \frac{\partial}{\partial x}+w \frac{\partial}{\partial z}
$$


is the material derivative. $\Delta$ is the operator defined by

$$
\Delta(f)=\delta^{2} \frac{\partial}{\partial x}\left(q \Lambda \frac{\partial f}{\partial x}\right)+\frac{\partial}{\partial z}\left(q \Lambda \frac{\partial f}{\partial z}\right) .
$$

the components of the work tensor are given by

$$
\begin{aligned}
& \pi_{x x}=2\left(\delta \tau_{x x} \frac{\partial u}{\partial x}+\tau_{x z} \frac{\partial u}{\partial z}\right), \quad \pi_{x z}=\delta^{2} \tau_{x x} \frac{\partial w}{\partial x}+\tau_{z z} \frac{\partial u}{\partial z}, \\
& \pi_{z z}=2\left(\delta^{2} \tau_{x z} \frac{\partial w}{\partial x}+\delta \tau_{z z} \frac{\partial w}{\partial z}\right)
\end{aligned}
$$

and

$$
R=\left[\delta \frac{\partial(q \Lambda)}{\partial x}\right]^{2}+\left[\frac{\partial(q \Lambda)}{\partial z}\right]^{2}
$$

The bottom boundary conditions are

$$
\begin{aligned}
& u \rightarrow s\left\{1+\frac{\beta}{\kappa} \ln (z)\right\}, \quad w \rightarrow 0, \quad \tau_{x x} \rightarrow-2^{3 / 2} s^{2}, \\
& \tau_{x z} \rightarrow|s| s, \quad \tau_{z z} \rightarrow-2^{1 / 2} s^{2}, \quad q \rightarrow 2^{5 / 4}|s|, \quad \Lambda \rightarrow c_{5} z,
\end{aligned}
$$

as $z \rightarrow 0$. To express the boundary conditions at the free surface, define $x^{\prime}$ and $z^{\prime}$ as coordinates parallel and perpendicular to the free surface, and $M$ as $\delta(\partial h / \partial x)$. Then the stress components $\tau_{x x}^{\prime}$ and $\tau_{z z}^{\prime}$ are

$$
\begin{aligned}
& \tau_{x x}^{\prime}=\left(\tau_{x x}+2 M \tau_{x z}+M^{2} \tau_{z z}\right) /\left(1+M^{2}\right), \\
& \tau_{z z}^{\prime}=\left(\tau_{z z}-2 M \tau_{x z}+M^{2} \tau_{x x}\right) /\left(1+M^{2}\right),
\end{aligned}
$$

and the free surface boundary conditions (2.11) become

$$
\begin{aligned}
& \tau_{x z}=\frac{M}{1-M^{2}}\left(\tau_{x x}-\tau_{x z}\right), \quad p=\frac{h}{F^{2}}+\beta^{2} \tau_{z z}^{\prime}, \quad w=\frac{\partial h}{\partial t}+u \frac{\partial h}{\partial x}, \\
& \left\{\frac{\partial}{\partial z}-\delta M \frac{\partial}{\partial x}\right\}\left\{\tau_{x x}^{\prime}, \tau_{z z}^{\prime}, q, \Lambda\right\}=0,
\end{aligned}
$$

at $z=h(x, t)$.

\section{Flow outside bore regions}

In accord with the scaling argument given in Section 1, we restrict our attention to the case $\beta \ll 1$, with the parameter $\sigma=\beta^{2} / \delta$ regarded as an $O(1)$ quantity. It is convenient in this section to use the coordinate $\zeta$ and the 
dependent variable $\lambda$ defined by

$$
\zeta=\frac{z}{h}, \lambda=\frac{\Lambda}{h},
$$

so that the flow domain is mapped into $|x|<\infty, 0<\zeta<1$.

Omitting $O\left(\beta^{2}\right)$ terms, we find from the vertical momentum equation and the second of the boundary conditions (2.30) that $p$ is independent of $\zeta$ and is given by $h / F^{2}$. Then, defining $d / d t$ by

$$
\frac{d}{d t}=\frac{\partial}{\partial t}+u \frac{\partial}{\partial x},
$$

the remaining equations of the model can be approximated by

$$
\begin{aligned}
& h \frac{\partial u}{\partial x}-\zeta \frac{\partial h}{\partial x} u+\frac{\partial w}{\partial \zeta}=0, \\
& h \frac{d u}{d t}+\left(w-\zeta \frac{d h}{d t}\right) \frac{\partial u}{\partial \zeta}+\frac{h}{F^{2}} \frac{\partial h}{\partial x}=\sigma\left(\frac{\partial \tau_{x z}}{\partial \zeta}+h\right), \\
& \beta c_{1} \frac{\partial}{\partial \zeta}\left(q \lambda \frac{\partial \tau_{x z}}{\partial \zeta}\right)=\beta \frac{q \tau_{x z}}{\lambda}+\tau_{z z} \frac{\partial u}{\partial \zeta}, \\
& \beta c_{1} \frac{\partial}{\partial \zeta}\left(q \lambda \frac{\partial \tau_{z z}}{\partial \zeta}\right)=\beta \frac{q}{\lambda}\left(\tau_{z z}+\frac{q^{2}}{4}\right), \\
& \beta c_{1} \frac{\partial}{\partial \zeta}\left(q^{2} \lambda \frac{\partial q}{\partial \zeta}\right)=\beta \frac{q^{3}}{8 \lambda}-\tau_{x z} \frac{\partial u}{\partial \zeta}, \\
& \beta c_{1} \frac{\partial}{\partial \zeta}\left(q \lambda \frac{\partial \lambda}{\partial \zeta}\right)=\beta\left\{\frac{c_{4}}{q}\left[\frac{\partial(q \lambda)}{\partial \zeta}\right]^{2}-c_{2} q\right\}+\frac{c_{3} \lambda}{q^{2}} \tau_{x z} \frac{\partial u}{\partial \zeta} .
\end{aligned}
$$

The boundary conditions are

$$
\begin{aligned}
& u \rightarrow s\left\{1+\frac{\beta}{\kappa} \ln (h \zeta)\right\}, \quad w \rightarrow 0, \quad \tau_{x z} \rightarrow|s| s, \\
& \tau_{z z} \rightarrow-2^{1 / 2} s^{2}, \quad q \rightarrow 2^{5 / 4}|s|, \quad \lambda \rightarrow c_{5} \zeta,
\end{aligned}
$$

as $\zeta \rightarrow 0$, and

$$
w=\frac{d h}{d t}, \quad \tau_{x z}=0, \quad \frac{\partial}{\partial \zeta}\left\{\tau_{z z}, q, \lambda\right\}=0,
$$

at $\zeta=1$. Here the equation and boundary conditions for $\tau_{x x}$ are uncoupled from the other equations, and are omitted.

We now expand the dependent variables in perturbation series of the form

$$
u=u^{(0)}+\beta u^{(1)}+\beta^{2} u^{(2)}+\cdots,
$$


and substitute into the governing equations. From (3.5), (3.7), and (3.8), we find that $u^{(0)}$ is independent of $\zeta$, and from the first of the boundary conditions (3.9) that $s^{(0)}=u^{(0)}$. Also, (3.3) implies that

$$
w^{(0)}=-\zeta h^{(0)} \frac{\partial u^{(0)}}{\partial x}
$$

and (3.4) that the lowest order contribution to $\tau_{x z}$ is linear in $\zeta$. The boundary conditions on $\tau_{x z}$ then imply that

$$
\tau_{x z}^{(0)}=(1-\zeta)\left|u^{(0)}\right| u^{(0)}
$$

Substituting (3.12) into the $O(1)$ version of the first boundary condition in (3.10) and (3.13) into the $O(1)$ version of the horizontal momentum equation (3.4) yields

$$
\begin{aligned}
& \frac{\partial h^{(0)}}{\partial t}+\frac{\partial}{\partial x}\left(h^{(0)} u^{(0)}\right)=0, \\
& \frac{\partial u^{(0)}}{\partial t}+u^{(0)} \frac{\partial u^{(0)}}{\partial x}+\frac{1}{F^{2}} \frac{\partial h^{(0)}}{\partial x}=\sigma\left\{1-\frac{\left|u^{(0)}\right| u^{(0)}}{h^{(0)}}\right\},
\end{aligned}
$$

a dimensionless version of the hydraulic equations (1.1) and (1.2). The lowest order approximation to the solution has now been determined, and the consistency of the approximation scheme is shown by the calculation of the $O(\beta)$ terms which follows.

The boundary conditions needed to determine these terms are

$$
\begin{aligned}
& u^{(1)} \rightarrow s^{(1)}+\frac{u^{(0)}}{\kappa} \ln \left(h^{(0)} \zeta\right), \quad w^{(1)} \rightarrow 0, \quad \tau_{z z}^{(0)} \rightarrow-2^{1 / 2}\left(u^{(0)}\right)^{2}, \\
& q^{(0)} \rightarrow 2^{5 / 4}\left|u^{(0)}\right|, \quad \lambda^{(0)} \rightarrow c_{5} \zeta, \quad \tau_{x z}^{(1)} \rightarrow 2\left|u^{(0)}\right| s^{(1)},
\end{aligned}
$$

as $\zeta \rightarrow 0$, and

$$
\begin{aligned}
& w^{(1)}=\frac{\partial h^{(1)}}{\partial t}+u^{(0)} \frac{\partial h^{(1)}}{\partial x}+u^{(1)} \frac{\partial h^{(0)}}{\partial x}, \quad \tau_{x z}^{(1)}=0, \\
& \frac{\partial}{\partial \zeta}\left\{\tau_{z z}^{(0)}, q^{(0)}, \lambda^{(0)}\right\}=0,
\end{aligned}
$$

at $\zeta=1$. The field equations become

$$
w^{(1)}=\zeta\left\{u^{(1)} \frac{\partial h^{(0)}}{\partial x}-h^{(1)} \frac{\partial u^{(0)}}{\partial x}\right\}-\frac{\partial}{\partial x}\left\{h^{(0)} \int_{0}^{\zeta} u^{(1)}\left(x, \zeta^{\prime}, t\right) d \zeta^{\prime}\right\},
$$

which is obtained by integrating the $O(\beta)$ continuity equation over $\zeta$, the 
$O(\beta)$ momentum equation

$$
\frac{\partial u^{(1)}}{\partial t}+\frac{\partial}{\partial x}\left\{u^{(0)} u^{(1)}+\frac{h^{(1)}}{F^{2}}\right\}=\frac{\sigma}{h^{(0)}}\left\{\frac{\partial \tau_{x z}^{(1)}}{\partial \zeta}+\frac{h^{(1)}}{h^{(0)}}\left|u^{(0)}\right| u^{(0)}\right\}
$$

and the $O(\beta)$ versions of [3.5]-(3.8).

The latter set of equations can be expressed in canonical form by noting that $\lambda^{(0)}$ depends only on $\zeta$ and by defining functions $f(\zeta), \sigma_{z z}(\zeta)$, and $\theta(\zeta)$ through

$$
\begin{aligned}
& u^{(1)}=s^{(1)}+\frac{u^{(0)}}{\kappa}\left\{\ln \left(h^{(0)}\right)+\kappa f(\zeta)\right\}, \\
& \tau_{z z}^{(0)}=\left(u^{(0)}\right)^{2} \sigma_{z z}(\zeta), \quad q^{(0)}=\left|u^{(0)}\right| \theta(\zeta) .
\end{aligned}
$$

Then, omitting the superscript on $\lambda$ and defining $F(\zeta)$ through

$$
F(\zeta)=\frac{1}{\sigma_{z z}}\left\{c_{1} \frac{d(\theta \lambda)}{d \zeta}+\frac{\theta}{\lambda}(1-\zeta)\right\}
$$

the $O(\beta)$ versions of (3.5)-(3.8) take the form

$$
\begin{aligned}
& \frac{d f}{d \zeta}=-F, \\
& c_{1} \frac{d}{d \zeta}\left(\theta \lambda \frac{d \sigma_{z z}}{d \zeta}\right)=\frac{\theta}{\lambda}\left(\sigma_{z z}+\frac{\theta^{2}}{4}\right), \\
& c_{1} \frac{d}{d \zeta}\left(\theta^{2} \lambda \frac{d \theta}{d \zeta}\right)=\frac{\theta^{3}}{8 \lambda}+(1-\zeta) F, \\
& c_{1} \frac{d}{d \zeta}\left(\theta \lambda \frac{d \lambda}{d \zeta}\right)=-c_{2} \theta-\frac{c_{3} \lambda}{\theta^{2}}(1-\zeta) F+\frac{c_{4}}{\theta}\left[\frac{d(\theta \lambda)}{d \zeta}\right]^{2},
\end{aligned}
$$

with boundary conditions

$$
f \rightarrow \frac{1}{\kappa} \ln (\zeta), \quad \sigma_{z z} \rightarrow-2^{1 / 2}, \quad \theta \rightarrow 2^{5 / 4}, \quad \lambda \rightarrow c_{5} \zeta,
$$

as $\zeta \rightarrow 0$, and

$$
\frac{d}{d \zeta}\left\{\sigma_{z z}, \theta, \lambda\right\}=0
$$

at $\zeta=1$.

Equations (3.22) - (3.27) define a boundary value problem which can be cast in standard form as a system of first order differential equations. This was solved numerically using pseudospectral collocation [6] to obtain the 


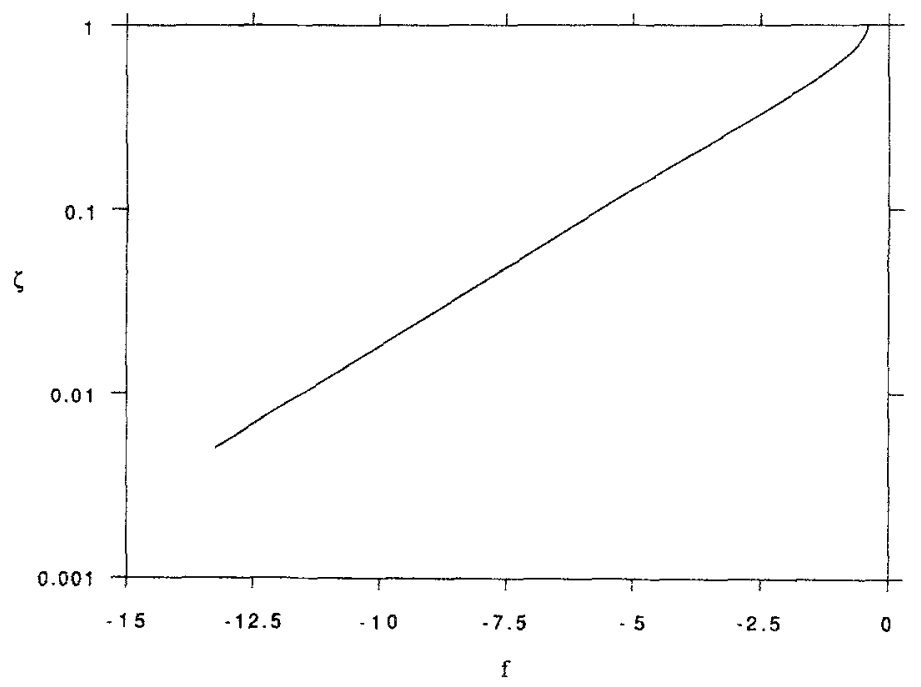

Figure 2

Function $f(\zeta)$.

graph of $f(\zeta)$ shown in Fig. 2 and the value

$$
\Gamma=\int_{0}^{1} f(\zeta) d \zeta=-2.330475
$$

for the integral of $f$. As can be seen, the variation of $f$ with $\zeta$ is approximately logarithmic over most of the depth of the fluid.

Returning now to (3.18)-(3.19), we evaluate (3.18) at $\zeta=1$, integrate (3.19) and the first equation in (3.20) over $\zeta$ from 0 to 1 , and introduce the definition

$$
I(x, t)=\int_{0}^{1} u^{(1)} d \zeta
$$

The result of this calculation is the set of equations

$$
\begin{aligned}
& \frac{\partial h^{(1)}}{\partial t}+\frac{\partial}{\partial x}\left(h^{(1)} u^{(0)}+h^{(0)} I\right)=0, \\
& \frac{\partial I}{\partial t}+\frac{\partial}{\partial x}\left(u^{(0)} I+\frac{h^{(1)}}{F^{2}}\right)=\sigma \frac{\left|u^{(0)}\right|}{h^{(0)}}\left\{\frac{h^{(1)} u^{(0)}}{h^{(0)}}-2 s^{(1)}\right\}, \\
& s^{(1)}=I-\frac{u^{(0)}}{\kappa}\left\{\ln \left(h^{(0)}\right)+\kappa \Gamma\right\},
\end{aligned}
$$

which can be solved numerically to determine $h^{(1)}, u^{(1)}$, and $s^{(1)}$. Similarly, higher order approximations can be obtained for the components of the Reynolds stress tensor and for $q$ and $\lambda$. As far as can be seen, the form 
assumed for the expansion (3.11) is correct, and the series is asymptotic in $\beta$.

It is convenient for computational purposes to combine the $O(1)$ and $O(\beta)$ equations as follows. Define $h(x, t)$ as the dimensionless depth and $\bar{u}(x, t)$ as the dimensionless depth-averaged velocity, and note that these are given here by

$$
\bar{u}=u^{(0)}+\beta I+O\left(\beta^{2}\right), \quad h=h^{(0)}+\beta h^{(1)}+O\left(\beta^{2}\right) .
$$

Then, also defining $\gamma(x, t)$ by

$$
\gamma=1-\frac{2 \beta}{\kappa}\{\ln (h)+\kappa \Gamma\}
$$

combining the $O(1)$ and $O(\beta)$ equations, and omitting terms $O\left(\beta^{2}\right)$, we obtain the system

$$
\begin{aligned}
& \frac{\partial h}{\partial t}+\frac{\partial(h \bar{u})}{\partial x}=0 \\
& \frac{\partial \bar{u}}{\partial t}+\bar{u} \frac{\partial \bar{u}}{\partial x}+\frac{1}{F^{2}} \frac{\partial h}{\partial x}=\sigma\left\{1-\gamma \frac{|\bar{u}| \bar{u}}{h}\right\} .
\end{aligned}
$$

When this system is solved, the velocity profile $u(x, \zeta, t)$ is given by

$$
u(x, \zeta, t)=\bar{u}(x, t)\{1+\beta[f(\zeta)-\Gamma)\},
$$

again with an $O\left(\beta^{2}\right)$ error. For the present problem, with the parameter range restricted as described in (1.5), the extensive calculations employed in [2] can be replaced by a simpler computation consisting of two steps, the solution of (3.35) and (3.36) to determine $h$ and $\bar{u}$, and the substitution of the solutions for $\bar{u}$ and $f(\zeta)$ into (3.37). The error in this procedure is formally $O\left(\beta^{2}\right)$, where, to repeat, typical values for $\beta$ are of order $10^{-1}$ or smaller.

Expressing (3.35)-(3.36) in dimensionless form gives a form of (1.1) and (1.2) in which the drag coefficient $C_{D}$ is expressed in terms of the dimensional depth $h_{d}$ by

$$
C_{D}=\beta^{2}\left\{1-\frac{2 \beta}{\kappa}\left[\ln \left(\frac{h_{d}}{H_{0}}\right)+\kappa \Gamma\right]\right\} \text {. }
$$

Equation (3.38) has the objectionable feature of depending explicitly on the characteristic depth scale $H_{0}$. However, if the small parameter $\varepsilon$ is defined by

$$
\varepsilon=\frac{\kappa}{\ln \left(h_{d} / z_{0}\right)},
$$


then

$$
\beta=\frac{\varepsilon}{1-(\varepsilon / \kappa) \ln \left(h_{d} / H_{0}\right)},
$$

and it can be verified that (3.38) becomes

$$
C_{D}=\varepsilon^{2}-2 \varepsilon^{3} \Gamma
$$

with an $O\left(\varepsilon^{4}\right)$, error, which is asymptotically equivalent to (3.38). Similarly, the dimensional velocity profile $u_{d}$ can be expressed in terms of the dimensional depth-averaged velocity $\bar{u}_{d}$ and the dimensional coordinate $z_{d}$ through

$$
u_{d}=\bar{u}_{d}\left\{1+\varepsilon\left[f\left(\frac{z_{d}}{h_{d}}\right)-\Gamma\right]\right\}
$$

which is asymptotically equivalent to (3.37) and which is independent of the characteristic depth.

According to the present theory, the first step in deriving a higher order version of the hydraulic equations requires correcting the value of the drag coefficient rather than inserting a factor multiplying the term $\bar{u}(\partial \bar{u} / \partial x)$ in (1.2), as is sometimes suggested in engineering texts. Furthermore, redoing the calculation using other second order turbulence models discussed in [7] shows that the only model dependent aspect of the calculation is the computation of the function $f(\zeta)$ and the constant $\Gamma$.

\section{Flow inside bore regions}

Because both (3.14) and (3.15) and the higher order approximation to the governing equations given by (3.35) and (3.36) are hyperbolic systems, they admit flow discontinuities in the form of bores. The flow in the bore regions cannot be treated using the hydraulic approximation developed in the previous section, and consequently an additional calculation is needed to determine the bore structure.

Experimentation with different possible length scales for a bore region suggests that the dimensional longitudinal extent of the region is of the order of the fluid depth. This in turn suggests introducing the dimensionless variables

$$
T=\frac{t}{\delta}, \quad X=\frac{x}{\delta}, \quad Z=z, \quad W=\delta w,
$$

to describe the flow in a bore region centered at $x=0$. The governing equations then become a version of $(2.16)-(2.31)$ in which $(x, z, t, w)$ are replaced by $(X, Z, T, W)$, and $\delta$ by unity. In particular, the horizontal 
momentum equation becomes

$$
\frac{\partial u}{\partial T}+u \frac{\partial u}{\partial X}+W \frac{\partial u}{\partial Z}+\frac{\partial p}{\partial X}=\beta^{2}\left\{\frac{\partial \tau_{x x}}{\partial X}+\frac{\partial \tau_{x z}}{\partial Z}+1\right\},
$$

where, as before, $\beta$ is a small parameter.

The right side of (4.2) must enter into the momentum balance in bore regions, and this can occur only if the stress components are $O\left(\beta^{-2}\right)$ in magnitude or if the vertical length scale is $O\left(\beta^{2}\right)$. The first possibility is incompatible with the boundary conditions $(2.28)$ because it implies an $O\left(\beta^{-1}\right)$ longitudinal velocity component, and the second implies $O\left(\beta^{2}\right)$ vertical velocity magnitudes throughout a bore region, in disagreement with visual observations of bores and hydraulic jumps. The present theory therefore suggests a double deck structure for the bore regions consisting of a bottom boundary layer of transverse dimensional thickness $O\left(\beta^{2} H_{0}\right)$ and an outer region of thickness $O\left(H_{0}\right)$, all within a region of longitudinal extent $O\left(H_{0}\right)$.

To describe the flow in the main body of the fluid, we introduce the additional definitions

$$
U=u, \quad P=p, \quad H=h, \quad T_{i j}=\beta^{2} \tau_{i j}, \quad Q=\beta q, \quad L=\Lambda, \quad \Pi_{i j}=\beta^{2} \pi_{i j},
$$

and substitute (4.1) and (4.3) into the dimensionless equations of Section 2. The horizontal momentum equation becomes

$$
\frac{\partial U}{\partial T}+U \frac{\partial U}{\partial X}+W \frac{\partial U}{\partial Z}+\frac{\partial P}{\partial X}=\frac{\partial T_{x x}}{\partial X}+\frac{\partial T_{x z}}{\partial Z}+\beta^{2},
$$

and the other field equations and the free surface boundary conditions are obtained by replacing $\tau_{i j}$ by $T_{i j}, \Lambda$ by $L$, the other variables by upper case symbols, and the parameters $\delta$ and $\beta$ by unity. The only small term in the equations describing the outer part of a bore region is the constant $\beta^{2}$ on the right side of (4.4), which represents the component of the gravity force in the longitudinal direction.

In expressing the equations governing the flow in the boundary layer region, we define the boundary layer variables

$$
\begin{aligned}
T & =\frac{t}{\delta}, \quad X=\frac{x}{\delta}, \quad \zeta=\frac{z}{\beta^{2}}, \quad \mu=u, \quad \omega=\frac{\delta}{\beta^{2}} w, \quad \pi=p, \\
t_{i j} & =\tau_{i j}, \quad \theta=q, \quad \lambda=\frac{\Lambda}{\beta^{2}}, \quad \sigma=s,
\end{aligned}
$$

where $\zeta, \theta, \sigma$, and $\lambda$ are not to be confused with their definitions in Section 
3. With $O\left(\beta^{2}\right)$ terms omitted, the boundary layer equations become

$$
\begin{aligned}
& \frac{\partial \mu}{\partial X}+\frac{\partial \omega}{\partial \zeta}=0, \\
& \frac{\partial \mu}{\partial T}+\mu \frac{\partial \mu}{\partial X}+\omega \frac{\partial \mu}{\partial \zeta}+\frac{\partial \pi}{\partial X}=\frac{\partial t_{x z}}{\partial \zeta}, \\
& \frac{\partial \pi}{\partial \zeta}=0, \\
& \beta\left\{c_{1} \frac{\partial}{\partial \zeta}\left(\theta \lambda \frac{\partial t_{x x}}{\partial \zeta}\right)-\frac{\theta}{\lambda}\left(t_{x x}+\frac{\theta^{2}}{4}\right)\right\}=2 t_{x z} \frac{\partial \mu}{\partial \zeta}, \\
& \beta\left\{c_{1} \frac{\partial}{\partial \zeta}\left(\theta \lambda \frac{\partial t_{x z}}{\partial \zeta}\right)-\frac{\theta}{\lambda} t_{x z}\right\}=t_{z z} \frac{\partial \mu}{\partial \zeta}, \\
& \beta\left\{c_{1} \frac{\partial}{\partial \zeta}\left(\theta \lambda \frac{\partial t_{z z}}{\partial \zeta}\right)-\frac{\theta}{\lambda}\left(t_{z z}+\frac{\theta^{2}}{4}\right)\right\}=0, \\
& \beta\left\{c_{1} \frac{\partial}{\partial \zeta}\left(\theta^{2} \lambda \frac{\partial \theta}{\partial \zeta}\right)-\frac{\theta^{3}}{8 \lambda}\right\}=-t_{x z} \frac{\partial \mu}{\partial \zeta}, \\
& \beta\left\{c_{1} \frac{\partial}{\partial \zeta}\left(\theta \lambda \frac{\partial \lambda}{\partial \zeta}\right)+c_{2} \theta-\frac{c_{4}}{\theta}\left[\frac{\partial(\theta \lambda)}{\partial \zeta}\right]^{2}\right\}=\frac{c_{3} \lambda}{\theta^{2}} t_{x z} \frac{\partial \mu}{\partial \zeta},
\end{aligned}
$$

with boundary conditions

$$
\begin{aligned}
& \mu \rightarrow \sigma\left\{1+\frac{\beta}{\kappa}[\ln \zeta-2 \ln (1 / \beta)]\right\}, \quad \omega \rightarrow 0, \quad t_{x x} \rightarrow-2^{3 / 2} \sigma^{2}, \\
& t_{x z} \rightarrow|\sigma| \sigma, \quad t_{z z} \rightarrow-2^{1 / 2} \sigma^{2}, \quad \theta \rightarrow 2^{5 / 4}|\sigma|, \quad \lambda \rightarrow c_{5} \zeta,
\end{aligned}
$$

as $\zeta \rightarrow 0$.

The equations in both regions are treated by expanding the dependent variables in series of the form

$$
\mu=\mu^{[0]}+\beta \ln (1 / \beta) \mu^{[1]}+\beta \mu^{[2]}+\cdots
$$

It can be seen by inspection that the lowest order contributions to $\mu$ and $\pi$ are independent of $\zeta$, and that matching with the outer solution yields

$$
\mu^{[0]}=U_{b}(X, T), \quad \pi^{[0]}=P_{b}(X, T),
$$

where $U_{b}$ and $P_{b}$ are the lowest order outer solutions for $U$ and $P$ evaluated at $Z=0$. Then, using the $O(1)$ contributions to the first boundary condition in (4.14), the continuity equation, and the horizontal momentum equation, we find that the lowest order solutions for $\sigma, \omega$, and $t_{x z}$ can be expressed in the form

$$
\sigma^{[0]}=U_{b}, \quad \omega^{[0]}=-\zeta \frac{\partial U_{b}}{\partial X}, \quad t_{x z}^{[0]}=a_{x z} B^{2} \zeta+\left|U_{b}\right| U_{b}
$$


where $a_{x z}$ is a constant and where $B(X, T)$ is the positive solution of

$$
a_{x z} B^{2}=\frac{\partial U_{b}}{\partial T}+U_{b} \frac{\partial U_{b}}{\partial X}+\frac{\partial P_{b}}{\partial X} .
$$

The $O[\beta \ln (1 / \beta)]$ contributions to $\mu$ and $\pi$ are also independent of $\zeta$, and, using (4.10), the $O(\beta)$ contribution to $\partial \mu / \partial \zeta$ is given by

$$
\frac{\partial \mu^{[2]}}{\partial \zeta}=\frac{1}{t_{z z}^{[0]}}\left\{c_{1} a_{x z} B^{2} \frac{\partial}{\partial \zeta}\left(\theta^{[0]} \lambda^{[0]}\right)-\frac{\theta^{[0]}}{\lambda^{[0]}}\left(a_{x z} B^{2 \zeta}+\left|U_{b}\right| U_{b}\right\} .\right.
$$

Substituting this equation into (4.9) and (4.11)-(4.13) yields four second order ordinary differential equations for the $O(1)$ contributions to $t_{x x}, t_{z z}, \theta$, and $\lambda$, with boundary conditions

$$
\begin{aligned}
& t_{x x}^{[0]} \rightarrow-2^{3 / 2}\left(U_{b}\right)^{2}, \quad t_{z z}^{[0]} \rightarrow-2^{1 / 2}\left(U_{b}\right)^{2}, \\
& \theta^{[0]} \rightarrow 2^{5 / 4}\left|U_{b}\right|, \quad \lambda^{[0]} \rightarrow c_{5} \zeta .
\end{aligned}
$$

If matching conditions, the constant $a_{x z}$, and the lowest order outer solution are known, this system can be solved using the numerical scheme described in Section 3 to provide the lowest order boundary layer solution for all the dependent variables. In addition, integrating (4.19) gives a solution for the $O(\beta)$ contribution to $\mu$ up to an additive function of $X$ and $T$.

To obtain the matching conditions, we assume that the solutions for the $O(\beta)$ contribution to $\mu$ and for the $O(1)$ contributions to $t_{i j}, \theta$, and $\lambda$ are of the form $\zeta^{m} \ln ^{n}(\zeta)$ as $\zeta \rightarrow \infty$, where $m$ is determined by noting that $\zeta=Z / \beta^{2}$ and that matching with the outer solution requires the expressions for $t_{i j}, \theta$, and $\lambda$ to be $O\left(1 / \beta^{2}\right), O(1 / \beta)$, and $O\left(1 / \beta^{2}\right)$, respectively, at the outer edge of the boundary layer. Since it can be shown that logarithms are absent from the matching conditions at this order of the solution, the matching conditions take the form

$$
t_{i j}^{[0]} \rightarrow a_{i j} B^{2 \zeta}, \quad \theta^{[0]} \rightarrow B \zeta^{(1 / 2}, \quad \lambda^{[0]} \rightarrow b \zeta, \quad \mu^{[2]} \rightarrow 2 B c \zeta^{(1 / 2)},
$$

as $\zeta \rightarrow \infty$, where $b, c$, and the $a$ 's are constants. The solution for the constants is found to be

$$
\begin{aligned}
& b=\left\{\frac{c_{3}+8 c_{2}}{6\left(c_{1} c_{3}+3 c_{4}-2 c_{1}\right)}\right\}^{1 / 2} \cong 0.507, \quad a_{x x}=-\frac{1-3 b^{2} c_{1}}{2-3 b^{2} c_{1}} \cong-0.435, \\
& a_{x z}= \pm \frac{1}{2 \sqrt{2}} \frac{\left(1-6 b^{2} c_{1}\right)^{1 / 2}}{\left(2-3 b^{2} c_{1}\right)} \cong \pm 0.147, \quad a_{z z}=-\frac{1}{2\left(2-3 b^{2} c_{1}\right)} \cong-0.283, \\
& c= \pm \frac{\left(2-3 b^{2} c_{1}\right)\left(1-6 b^{2} c_{1}\right)^{1 / 2}}{(2 \sqrt{2}) b} \cong \pm 0.904,
\end{aligned}
$$

where $a_{x z}$ and $c$ have the same sign as the right side of (4.18). 
Working now to lowest order and expressing the matching conditions (4.21) in terms of the outer variables, the outer solution satisfies the matching conditions

$$
\begin{aligned}
& T_{x x}^{[0]} \rightarrow a_{x x} B^{2} Z, \quad T_{x z}^{[0]} \rightarrow a_{x z} B^{2} Z, \quad T_{z z}^{[0]} \rightarrow a_{z z} B^{2} Z, \\
& Q^{[0]} \rightarrow B Z^{1 / 2}, \quad L^{[0]} \rightarrow b Z, \quad W^{[0]} \rightarrow-Z \frac{\partial U_{b}}{\partial X},
\end{aligned}
$$

as $Z \rightarrow 0$, where, repeating earlier definitions,

$$
U_{b}=U^{[0]}(X, T, 0), \quad P_{b}=P^{[0]}(X, T, 0),
$$

and $B$ is the positive solution of (4.18). With superscripts omitted, the lowest order solution in the outer region solves these matching conditions, the continuity and momentum equations

$$
\begin{aligned}
& \frac{\partial U}{\partial X}+\frac{\partial W}{\partial Z}=0, \\
& \frac{\partial U}{\partial T}+U \frac{\partial U}{\partial X}+W \frac{\partial U}{\partial Z}+\frac{\partial P}{\partial X}=\frac{\partial T_{x x}}{\partial X}+\frac{\partial T_{x z}}{\partial Z}, \\
& \frac{\partial W}{\partial T}+U \frac{\partial W}{\partial X}+W \frac{\partial W}{\partial Z}+\frac{\partial P}{\partial Z}=\frac{\partial T_{x z}}{\partial X}+\frac{\partial T_{z z}}{\partial Z},
\end{aligned}
$$

a system of five parabolic equations for $Q, L$, and the components of the stress tensor, and the free surface boundary conditions. The field equations for the turbulence quantities and the free surface boundary conditions are obtained from (2.19) $-(2.23)$ and $(2.29)-(2.31)$ by making the substitutions given in the phrase following Eq. (4.4).

If $T_{x x}, T_{x z}$, and $T_{z z}$ were known, solution of (4.25)-(4.27) would require three boundary conditions, which are given here by the last equation in (4.23) and by the kinematic and dynamic boundary conditions at the free surface. Solving the system of equations for $Q, L$, and the $T$ 's requires ten additional boundary conditions. These are given by the first equation in (2.30), the four equations in (2.31), and the five equations obtained by eliminating $B$ between (4.18) and (4.23). The lateral boundary conditions on the components of the stress tensor and on $Q$ are

$$
T_{x x} \rightarrow 0, \quad T_{x \dot{z}} \rightarrow 0, \quad T_{z z} \rightarrow 0, \quad Q \rightarrow 0,
$$

as $|X| \rightarrow \infty$, and the other variables match in this limit with the solution given by the hydraulic approximation of Section 3 .

We will not attempt a numerical solution for the flow in the outer part of bore regions in the present study, but it appears that the problem describing the lowest order approximation is well set in the sense that the correct number of boundary conditions is available. Assuming that a 
solution for the bore structure does exist, jump conditions relating the flow on two sides of a discontinuity predicted by the hydraulic equations are obtained by integrating (4.25) and (4.26) over an area bounded below by $Z=0$, above by $Z=H(X, T)$, and on the left and right by

$$
X=X_{-} \equiv-A+C T, \quad X=X_{+} \equiv A+C T,
$$

where $A$ and $C$ are constants.

Letting $A$ tend to $\infty$, noting that the solution for $U$ at the outer edges of a bore region is independent of $Z$ to lowest order in $\beta$, and omitting details of the derivation, we find that for a bore moving at constant speed $C$ the outer solution satisfies the jump conditions

$$
[H U]=C[H], \quad\left[H U^{2}+\frac{1}{2 F^{2}} H^{2}\right]=C[H U],
$$

where, as before, $[f]$ denotes the discontinuity in a function $f$ as determined by the hydraulics equations. Equation (4.30) is a dimensionless version of the classical jump conditions given by (1.3). By contrast, the theory given in [8], which attempts to resolve the bore structure by using a viscous type body force $v\left(\partial^{2} U / \partial X^{2}\right)$ in (4.26) in place of $\partial T_{x x} / \partial X$, yields a momentum jump condition in disagreement with the second equation in (1.3). In our view this occurs because the stress tensor employed in [8] is asymmetric, and is therefore incorrect.

\section{Discussion}

The present calculation is based on the assumptions that open channel flows can be treated using standard second order turbulence models, and that the equations of mathematical hydraulics can be obtained by a rational approximation process valid for a parameter range discussed in the text. The theory takes two forms, depending on whether a point in the fluid lies within or exterior to a bore region. The surface elevation and depthaveraged longitudinal velocity component outside bore regions are found to satisfy the classical hydraulics equations to lowest order in a perturbation expansion, and a version of these equations involving a variable bottom drag coefficient is derived by carrying the theory to higher order. The variation of the longitudinal velocity component with distance from the channel bottom and the lowest order solution for the Reynolds stress tensor and other turbulence quantities are also computed.

The flow within bore regions is found to have a double deck structure consisting of an outer region with characteristic longitudinal and transverse length scales of the order of the fluid depth, and a bottom boundary layer with a longitudinal scale of the order of the fluid depth and a transverse 
scale of the order of the depth times the drag coefficient. Equations and matching conditions for the flow in the two regions are derived, and it is shown that the laws of mass and momentum balance for the bore regions provide the classical jump conditions of mathematical hydraulics used to connect solutions valid on either side of a flow discontinuity.

As indicated in references [2], [5], and [7], enough experience has been gained in turbulence modelling to infer that thin layer flows of the type occuring outside bore regions are well described by standard second order turbulence models. Consequently, assuming that the analysis is correct, the theory given in Section 3 should provide accurate approximate equations for describing the flow outside bore regions. The theory of Section 4 on the bore structure is more tentative because it is not known how well the flow is described by second order turbulence models and because we have not provided a numerical solution for the equations governing the bore structure or a mathematical proof that a solution exists and is unique. Whether the problem has a physically meaningful solution is an open question which deserves further study because of the interest in bores in near-shore oceanography and other branches of applied fluid mechanics.

\section{References}

[1] G. B. Whitham, Linear and Nonlinear Waves, pp. 83-91. John Wiley \& Sons. New York 1974.

[2] W. Rodi, Turbulence Models and Their Application in Hydraulics - A State of the Art Review. International Association for Hydraulic Research, Delft, The Netherlands 1980.

[3] M. Van Dyke, Adv. Appl. Mech. 25, 1-45 (1987).

[4] S. J. Jacobs (submitted, 1990).

[5] W. S. Lewellen, in Handbook of Turbulence, Vol. 1. (Eds. W. Feast and T. H. Moulden), pp. 237-280. Plenum Press, New York 1977.

[6] S. J. Jacobs, J. Comp. Phys. (in press, 1990).

[7] B. E. Launder, In Theoretical Approaches to Turbulence (Eds. D. L. Dwoyer, M. Y. Hussaini and R. G. Voight), pp. 155-186. Springer, Berlin 1985.

[8] D. J. Needham and J. H. Merkin, Proc. R. Soc. Lond A, 394, 259-278 (1984).

\section{Summary}

The relation between classical hydraulics and modern turbulence modelling is discussed for the case of two-dimensional open channel flow down an inclined plane. A second order turbulence model describing the flow is treated asymptotically for the parameter range $F \geq O(1), \delta \ll 1, \beta \ll 1$, and $\delta=O\left(\beta^{2}\right)$, where $F$ is the Froude number, $\delta$ is the aspect ratio, and $\beta$ is the square root of a characteristic drag coefficient. The Chezy law formulation of mathematical hydraulics is derived as the lowest order approximation to the solution for the flow outside bore regions, and the transverse variation of the longitudinal velocity component is determined at the next stage of the analysis. It is shown that flow discontinuities calculated using the equations of mathematical hydraulics are resolved in bore regions of transverse length scale $O\left(H_{0}\right)$, where $H_{0}$ is the characteristic fluid depth. The bore structure is found to consist of a highly turbulent outer region with transverse length scale $O\left(H_{0}\right)$ in which the turbulence intensity is $O(1)$, and a bottom boundary layer of transverse length scale $O\left(\beta^{2} H_{0}\right)$, in which the turbulent stresses decrease rapidly to satisfy the bottom boundary conditions. The jump conditions of mathematical hydraulics at flow discontinuities are verified, and it is inferred that classical hydraulics provides an acceptable approximation to the flow outside bore regions for the parameter range considered in the theory. 